
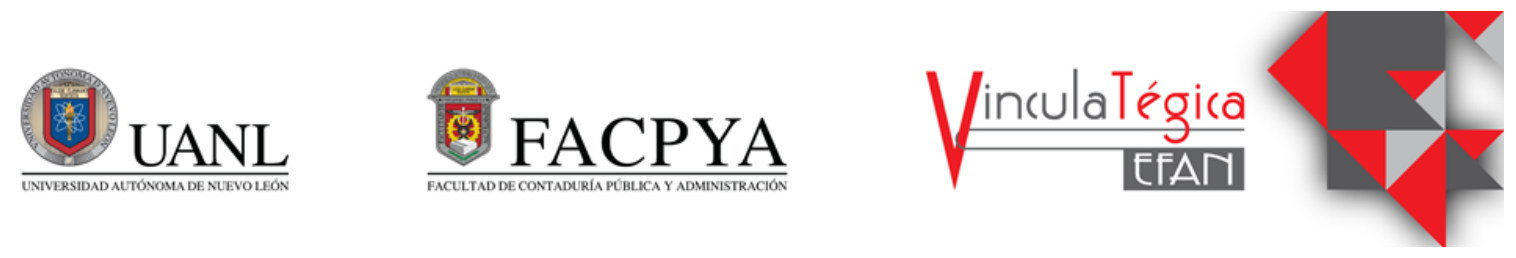

\title{
Gestión de Inventarios eficiente por la aplicación de Control Interno
}

\author{
Beatriz Seijas Rodríguez ${ }^{1}$ \\ ${ }^{1}$ Universidad Autónoma de Nuevo León, Facultad de Contaduría Pública y Administración, Av. Universidad \\ s/n, col.Ciudad Universitaria, Monterrey, Nuevo León, México, bseijasr@gmail.com, \\ (+52) 8135320404 \\ Información del artículo revisado por pares \\ Fecha de aceptación: junio-2021 \\ Fecha de publicación en línea: diciembre-2021 \\ DOI: https://doi.org/10.29105/vtga7.2-21:
}

\section{Resumen}

En el mundo organizacional, mantener un inventario para su venta o uso futuro en cadenas productivas, es una práctica común y necesaria. La variedad empresarial, en términos de objeto social, razón de ser, misión, visión y sus respectivos nichos de mercado; definen el posicionamiento de las organizaciones por categoría. De lo anterior, emergen las empresas de venta al menudeo, mayoristas, minoristas, productores, así como los distintos eslabones de las cadenas globales de valor. Sin embargo, el inventario, constituye su factor común, de ahí que administrarlo continúe siendo objeto de estudio. Su gestión eficiente está estrechamente relacionada con los procedimientos de control interno practicados, los cuales permitirán o no, alcanzar los objetivos del negocio. Por tanto, dentro de las normas reguladoras, se destaca como referente el Modelo de Control Interno COSO 2013, siendo el más empleado por las empresas que cotizan en las Bolsas de Valores de Estados Unidos

Palabras clave: Control Interno, inventarios, administración eficiente

\begin{abstract}
In the organizational world, keeping an inventory for sale or future use in productions chains is a common and nesessary practice. The business variety, in temrs of corporate purpose, reason for being, mission, vision and their respective market niches; define the positioning of organizations by category. From the above, the retail companies, wholesalers, retailers, producers, as well as a different links of the global value chains emerge. However, inventory is its common factor, hence managing it continues to be the object of study. Its efficient management is closely related to the internal control procedures practiced, which will make it possible or not to archieve the business objectives. Therefore, within the regulatory standards, the COSO 2013 Internal Control Model, stands out as a reference model, being the most used by companies listed on the United States Stock Excchanges.
\end{abstract}

Keywords: Internal Control, inventories, efficient management

JEL: M42 


\section{INTRODUCCIÓN}

En el entorno actual, complejo, dinámico y globalizado; las organizaciones deben sostener el control exhaustivo sobre los inventarios, ya sea de materias primas y/o productos terminados. De ahí que, el control de inventarios eficiente desempeñe un rol protagónico al respecto de la estabilidad operacional y la satisfacción sostenible de la demanda.

En el orden de las consideraciones anteriores, realizar eficazmente el control de inventarios, así como su seguimiento en tiempo real, ofrecen beneficios ineludibles, tales como: realizar pronósticos de demanda, minimizar costos y maximizar beneficios. Lo anterior se traduce en niveles óptimos de rentabilidad y saldos favorables en el flujo de efectivo.

Por tanto, las decisiones sobre los niveles de inventarios implicarán beneficios o gastos innecesarios sobre las inversiones de recursos financieros. De un lado, invertir más de lo necesario en discordancia con la capacidad operativa que permita su salida, deriva en inventario sobre stock, generando costos adicionales, asociados principalmente a su mantenimiento. De otro, invertir en valores inferiores a la demanda, implica, no satisfacerla, de ahí la pérdida del poder de mercado, entre otras consecuencias negativas en términos de utilidad.

Hechas las observaciones anteriores, se puede afirmar que, en el mundo organizacional, mantener un inventario para su venta o uso futuro es una práctica común y necesaria. Es evidente entonces, que, para multiplicar los niveles de rentabilidad, ha de llevarse a cabo la planificación y administración estratégica en aras de mantener alineados los objetivos de negocio con la misión y visión de las empresas.

Por tanto, para llevar a cabo el control eficiente de inventarios se sugiere el empleo del Marco de Control Interno COSO 2013, el cual define 3 objetivos de negocio: Operativos, de Información y de Cumplimiento y establece, a su vez, la implementación de sus 5 componentes y 17 principios para el logro de estos (Committee of Sponsoring Organizations of the Treadway Commission, 2013).

El modelo anterior empleado, de forma eficaz, resultaría una herramienta poderosa hacia el logro de los objetivos de negocio, los niveles de rentabilidad esperados, así como las metas trazadas al respecto de la eficiencia operativa. En este orden, el empleo del modelo mencionado en temas particulares de control eficiente de inventarios anuncia beneficios alcanzables y mejorables.

\section{OBJETIVO}

El objetivo de esta investigación de tipo descriptivo es determinar los beneficios mostrados en la gestión eficiente de inventarios a causa del empleo de controles internos fundamentados en el Modelo de Control Interno COSO 2013.

\section{MARCO TEÓRICO}

Para alcanzar el soporte teórico que sustente los beneficios brindados por la administración eficiente del inventario, en un primer momento se fundamenta y valida descriptivamente, la variable "inventarios", seguido de las teorías asociadas y los estudios empíricos que muestran la aplicación de sistemas de control de inventarios, dando lugar al nivel de aplicación del Marco de Control Interno COSO 2013 con relación a su trascendencia.

\subsection{Marco Teórico sobre la Gestión de Inventarios}

El inventario constituye un importante factor a controlar, representa uno de los activos más grandes e importantes de la organización. Su valor en libros se registra tanto en el Balance General como en el Estado de Resultados. Entre otras razones, se le atribuye un rol protagónico sobre la claridad y estabilidad operativa, pues permite conocer el valor de la mercancía vendida en un período o ejercicio determinado. De ahí que, se considere oportuno, describir a continuación, algunas definiciones relacionadas con la gestión de inventarios.

El Inventario consiste en un listado ordenado, detallado y valorado de los bienes de una empresa. (...) agrupando los que son 
similares y valorados, ya que se deben expresar en valor económico para que formen parte del patrimonio ${ }^{1}$ de la empresa. (Cruz, 2018)

De otro lado, el inventario (...) es la verificación y control de los materiales o bienes patrimoniales de la empresa, que realizamos para regularizar la cuenta de existencias contables con las que contamos en nuestros registros, para calcular si hemos tenido pérdidas o beneficios (Meana, 2017).

En el orden de las ideas anteriores, resulta oportuno precisar las variables que afectan la gestión de inventarios, tal como se describe en el siguiente epígrafe.

\subsubsection{Variables que afectan la gestión de inventarios}

El proceso de inventariar comprende desde la función de aprovisionamiento ${ }^{2}$ hasta la de distribución del producto.

Por tanto, dentro de la función de aprovisionamiento las variables que afectan la gestión de inventarios son: el tiempo, el cual comprende el tiempo de entrega del proveedor, el tiempo de realización del pedido, así como el tiempo de recepción en almacén; la demanda: donde su variación se produce con relación a su entorno y al volumen de comercialización del producto (dada su unidad de medida), en relación con las expectativas y al conocimiento del comportamiento futuro (estable o aleatorio), atendiendo a su implicación con el tiempo (homogénea o heterogénea) y en relación con la disponibilidad del producto (diferida o perpetua); los costos: dados por la gestión y tenencia de inventario, que generan gastos por conceptos de adquisición, almacenamiento y demanda no cubierta (Cruz, 2018). De todo lo anterior se deriva la importancia de revisar descriptivamente los objetivos y teorías de inventario.

\subsubsection{Objetivos del Inventario}

1 Conjunto de bienes, derechos y obligaciones futuras (de una empresa), siendo: los bienes las partidas tangibles e intangibles, los derechos sus bienes futuros y las obligaciones son derechos que tienen otros sobre la empresa. (Cruz, 2018)
Satisfacer las necesidades y expectativas de los clientes, debe encontrarse el equilibrio ideal, brindándoles el mayor nivel de servicios posible con el menor nivel de inventario. En relación con los principales objetivos de la gestión de stock se encuentran: apoyar la rentabilidad de la compañía, disminuir las ventas perdidas, entregar oportunamente, dar un nivel adecuado de servicio con un costo de stock en equilibrio y responder ante imprevistos de la demanda y la oferta (amortiguador) (Mora, 2016).

\subsubsection{Teorías de Inventario}

Las teorías alrededor del inventario surgen a raíz de la necesidad de alinear la satisfacción de la demanda con la minimización de costos por conceptos de inversión de capital, faltantes y almacenamiento. Precisando entonces, al respecto de las decisiones de inventario, han de responderse las siguientes interrogantes: ¿Cuánta mercancía solicitar? ¿Cuándo solicitarla?

Por tanto, atendiendo a la capacidad predictiva de la demanda, el inventario se puede clasificar en dos grupos como sigue: determinístico, cuando la demanda de períodos futuros puede ser pronosticada de forma precisa y estocástico o probabilístico, cuando el pronóstico a futuro de la demanda no puede ser determinado con exactitud.

En ese mismo sentido, resulta oportuno describir las principales teorías de inventarios. Entonces, según Lizarazo \& Pérez (2017) el Modelo Determinístico de Revisión Continua engloba diferentes situaciones alrededor del empleo de inventarios, de ellas la más usual y de fácil comprensión, radica en el agotamiento de existencias y su reposición, al estilo de una secuencia continua, que ha de presentar fallas si es interrumpida. Por otra parte, la versión anterior, si bien es sumamente sugerente, no es la única situación para desarrollar, de ahí que se muestren a continuación, otros escenarios

\footnotetext{
${ }^{2}$ Forma parte de la logística de una empresa y se encarga de las compras y de su gestión. (Cruz, 2018)
} 
que contempla el modelo determinístico de revisión continua.

\subsubsection{Modelo $\mathrm{EOQ}^{3}$ Básico}

El objetivo principal de este modelo es determinar la cantidad y la frecuencia de ordenar para minimizar los costos de inventario sin interrumpir la operatividad de la organización. Lo anterior, requiere el conocimiento de la demanda fija en determinada unidad de tiempo, para así calcular el punto de reorden ${ }^{4}$.

Para lo anterior, están dados los siguientes supuestos: tasa de demanda (d) dada en unidades de tiempo, conocida; la cantidad ordenada de lote (Q) para el reabastecimiento del inventario llega justo cuando sus niveles toman valor cero y no se permite la existencia de faltantes (Lizarazo \& Perez, 2017).

\subsubsection{Modelo EOQ con faltantes planeados}

En comparación con el modelo anterior que tiene como supuesto establecido la no permisión de faltantes, según Taha (2012) existen escenarios donde los costos de almacenamientos son elevados con relación a los costos por faltantes, en cuyo caso es necesario permitirse faltante, dando lugar a la creación de órdenes pendientes, que han de funcionar, si y solo si, los clientes están de acuerdo con añadir un retraso razonable a la recepción de sus pedidos.

Por tanto, los supuestos del modelo son: tasa de demanda (d) en unidad de tiempo, conocida, la cantidad ordenada de lote (Q) para el reabastecimiento del inventario llega justo cuando sus niveles toman valor cero y se permiten faltantes.

\subsubsection{Modelo EOQ con descuentos por cantidad}

Los modelos descritos anteriormente, sugieren un valor de producto constante, por lo que la diferencia clave que añade este modelo radica en la inclusión de los descuentos realizados

\footnotetext{
${ }^{3}$ Economic Order Quantity por sus siglas en inglés y Cantidad Económica de Pedido por sus siglas en español
}

por proveedores en relación con las cantidades compradas, que, a su vez permite optimizar los costos totales.

En este mismo orden, según Taha (2012) los supuestos de este modelo coinciden con los del Modelo EOQ básico con la siguiente adición: empleando la fórmula de cantidades óptimas del modelo EOQ básico, se llevará a cabo una aplicación para cantidad óptima por descuento ofrecido por el proveedor. Ahora bien, si el resultado obtenido sobre las cantidades óptimas no forma parte del rango de cada descuento, se aproximará al valor más cercano, adicionalmente, los costos totales, serán calculados por unidad de tiempo de cada descuento para minimizar la función.

\subsubsection{Elementos relevantes sobre las Teorías de Inventario}

Por sobre sus diferencias, las teorías anteriores, recalcan la necesidad de determinar eficientemente la cantidad y la frecuencia de pedidos que minimicen los costos de inventario, hasta su valor óptimo, sin afectar la operatividad de la empresa.

Con el tiempo, estas teorías han evolucionado, incorporando en sus ecuaciones variables más complejas en cuanto a medición e interpretación. Lo anterior, con la intención de emplear modelos robustos que simplifiquen la realidad acertadamente.

Ahora bien, las teorías descritas, no son más que herramientas a poner en práctica añadiendo la variable "ser humano". Por tanto, la resolución de las mismas, con la inclusión lógica de la variable mencionada, implica la presencia de error y, por ende, de riesgos. Es evidente entonces, la necesidad de establecer controles sobre los procedimientos, supervisar más que el cálculo de las variables incógnitas, velar porque otros elementos, como falta de control interno, no empañen los objetivos y metas por alcanzar.

\subsubsection{Administración eficiente de inventario. Estudios empíricos}

\footnotetext{
${ }^{4}$ Punto de Reorden $=($ Tasa de demanda $) x$ (Tiempo de entrega) (Lizarazo \& Perez, 2017)
} 
Sobre la base de las consideraciones anteriores, según Pérez et al. (2013) con la intención de minimizar costos y maximizar la eficiencia económica, una de las alternativas más influyentes a considerar es, la aplicación de un Sistema de Gestión de Inventarios. ¿Por qué?, pues porque incrementa los niveles de servicio al cliente, traducido en un indicador de satisfacción, dado en gran medida por los pedidos atendidos en un período de tiempo "X". Es evidente entonces, el aumento progresivo de la liquidez hacia niveles que faciliten la obtención del punto óptimo de seguridad, con la intención de anticipar y/o prevenir fluctuaciones de la demanda, logrando estabilizar los inventarios necesarios.

De esta forma, la aplicación de un sistema u otro, de gestión de inventarios podría generar o no, un problema de toma de decisiones enfocado principalmente en la siguiente interrogante: ¿cuánto producir o adquirir? Equivocar en la decisión podría provocar la reducción del inventario e interpretarse a priori como un evento satisfactorio, por la lógica reducción de la inversión; sin embargo, el riesgo de insatisfacción de la demanda está presente, siendo causa posible de un cuello de botella en las operaciones de la empresa. Precisando de una vez, la gestión eficiente de inventarios permite determinar el valor óptimo respecto al nivel de producto que debe mantenerse.

Por tanto, los conceptos anteriores pueden acotarse como la eficiente planificación del nivel de stock $^{5}$ necesario y suficiente, para que la empresa haga frente a la demanda en el corto y mediano plazos, que tribute al correcto y continuo funcionamiento de los procesos de producción y ventas. Por ende, para elevar los niveles de eficiencia, la administración debe favorecer la gestión de inventarios.

Por su parte, Lopes \& Gómez (2012) realizan una investigación basada en una auditoría logística que pretende evaluar el nivel de gestión de inventarios de las empresas. ¿Cómo lo hacen? Se centran en los

5 (concepto anglosajón que se refiere a las existencias) aquellos bienes o productos de la aspectos organizacionales para definir una estrategia de control que conduzca su investigación hacia el planteamiento de su propuesta “¿cómo realizar la evaluación sobre la gestión de inventarios?". Lo anterior, está sustentado en el siguiente supuesto: los modelos matemáticos de investigación de operaciones para la gestión de inventarios no son del todo efectivos. Finalmente, la investigación permite conocer la tendencia creciente de las publicaciones que analizan los modelos de gestión colaborativos para gestionar el inventario. Lo anterior resulta provechoso, pues se entrelazan de forma integrada todas las áreas y procesos de la logística, aportando información relevante para la mejora continua de la gestión del inventario. Por tanto, no se desestima el empleo de modelos matemáticos, sin embargo, no pueden estar divorciados de los modelos de gestión colaborativos. En ese sentido, proponen el diseño de una herramienta, que aplique el principio de parsimonia, para la evaluación del nivel de gestión organizacional del inventario a nivel de la empresa y la auditoría de los procesos que en dicha gestión intervienen.

De otro lado, López (2011) se propone diseñar un sistema de control interno eficiente, mediante el cual el ciclo del inventario sea asegurado, agilizado y no genere costos adicionales. La metodología empleada consiste básicamente en la revisión bibliográfica de los exponentes del tema considerados, así como la aplicación de una encuesta al gerente, empleados y clientes. Se evalúan diferentes criterios de eficacia y eficiencia respecto al ciclo de inventarios. Emplea un análisis de frecuencias estadísticas mediante la distribución $X^{2}$, como estadístico de prueba, para analizar la representatividad de las frecuencias y la verificación de su $H_{0}$ "El control interno ineficiente al ciclo de inventarios, no conlleva a una disminución en la rentabilidad de la Ferretería Ángel López ubicada en la ciudad de Ambato". Finalmente, se rechaza la $H_{0}$ y no se rechaza la $H_{1}$ "El

empresa que necesitan ser almacenados para su posterior venta o incorporación al proceso de fabricación. (Cruz, 2018) 
control interno ineficiente al ciclo de inventarios, conlleva a una disminución en la rentabilidad de la Ferretería Ángel López ubicada en la ciudad de Ambato." Por tanto, se definen las siguientes recomendaciones: aplicar el modelo COSO para evaluar el ciclo de inventarios, aplicar el modelo de inventarios conocido como cantidad económica de pedido, implementar políticas que permitan mejorar las actividades que se cumplen en la empresa, mantener actualizado el control contable de la empresa, en especial en todas las cuentas relacionadas con el rubro de inventarios.

Luego, Morocho (2015) investiga los problemas inherentes del control interno en la empresa ecuatoriana ORODIESEL, los que recaen en la gestión de inventarios, y afirma su relación directamente proporcional sobre la rentabilidad de la organización. Por lo anterior, se propone implementar una guía de control interno para los inventarios y cumple su objetivo, empleando la siguiente metodología: observación y realización de entrevistas. En el orden de las consideraciones anteriores, dentro de los principales problemas detectados, resalta la inexistencia de un bodeguero por lo que sus funciones recaen en el gerente, asimismo en el proceso de adquisición de productos, autorizado solo por el contador, no media una orden de compra, y solo se evalúan las ofertas de a lo sumo tres proveedores. De forma general carecen de normas claras y sostenibles para el control de inventarios. Demostrado lo anterior, así como el estado de situación financiera de la empresa en función de sus pruebas corriente, ácida, de liquidez, etc., el autor corrobora la relación directa del control de inventarios respecto al comportamiento de la rentabilidad.

\subsubsection{Trascendencia del Control Interno}

¿Qué se entiende sobre Control Interno? COSO lo define como un proceso, ejecutado por la junta de directores, la administración principal y otro personal de la entidad, diseñado para proveer seguridad razonable en relación con el logro de los objetivos de la organización (Mantilla, 2018).

Por tanto, el Control Interno, representa el componente integral de una empresa, aquella que facilita y eficientiza los procesos para su desarrollo en su macro y microentorno, de ahí que, la organización debe practicar cierto grado de control sobre todos los procesos del negocio, acentuado en aquellos elementos que influyen potencialmente en su desempeño. Lo anterior, añadido a los controles de tipo preventivos y aplicado a la gestión de inventarios, contribuirá positivamente en el correcto, transparente y previo ejercicio de dicha gestión, por lo cual merece especial atención dentro de esta investigación.

\subsubsection{Control Interno. Objetivos y su relevancia en el tratamiento de los inventarios}

Tales objetivos son: eficacia y eficiencia de las operaciones; confiabilidad de la información financiera; cumplimiento de normas y obligaciones; y salvaguarda de activos (Mantilla, 2018).

Ahora bien, el control interno dirigido a la gestión eficiente de inventarios es altamente significativo, ya que permite generar reportes financieros confiables. De ahí que, al respecto de dicha gestión, al control interno se le atribuyen ciertas funciones reguladoras, mismas que abarcan desde el método de clasificación, el conjunto de reglas admitidas en la organización para resguardar los activos, hasta la comprobación de la transparencia, autenticidad y efectividad de los procedimientos administrativos y financieros. Todo lo anterior, con la intención de potenciar eficientemente el cumplimiento de las metas y objetivos proyectados.

\subsubsection{Relevancia del Modelo de Control Interno COSO 2013}

Ahora bien, se han publicado diversos modelos de Control. Los modelos más conocidos son: el COSO (USA), el Vienot (Francia), el Peters (Holanda), King (Sudáfrica) y MICIL (adaptación del COSO para Latinoamérica) (Rivas, 2011).

Precisando de una vez, en relación con las bases citadas en el Marco de Control Interno COSO 2013, los sistemas de control interno en la organización fungen como herramienta para el logro de los objetivos de 
negocio, esto, vinculado al orden que se pretende de a la operación de las empresa, dicho Marco de Control Interno, establece que las organizaciones deben adoptar su misión y visión, definir estrategias, fijar sus objetivos y planes para lograrlos, mismos que pudieran ser afectados por amenazas o riesgos en la operación, sugiriendo la implementación de los 5 componentes y 17 principios, de dicho Marco para el logro de los objetivos de Negocio (Committee of Sponsoring Organizations of the Treadway Commission, 2013).

En este mismo orden, los sistemas de control interno de acuerdo con las bases del Marco de Control Interno COSO 2013, tienen un rol importante para ordenar esfuerzos y recursos establecidos para cumplir los objetivos de Negocio. Sin embargo, factores externos, desapego a valores organizacionales, brechas de control en sistemas de información, entre otros, pudieran ocasionar que el personal violente las Políticas y Procedimientos de las Organizaciones y afectar el cumplimiento de los objetivos de Negocio (Barrera, 2020).

Entonces, el modelo de Control Interno COSO 2013, ofrece un acertado sistema de regulaciones, por medio de un adecuado ambiente de control, eficaz evaluación de riesgos, actividades de control ordenadas, información y comunicación permanentes y monitoreo constantes, acciones estas, que, en su conjunto, permitirá mejorar la gestión de inventarios en la empresa. Los criterios anteriores, apoyan que el Marco de Control Interno COSO 2013 funja como base para la implementación de una normativa que guíe a la administración en el proceso de manejo eficaz de riesgos, hacia el logro de los objetivos de negocio y particularmente eficientizar la gestión de inventarios.

\subsubsection{Aplicabilidad del Modelo de Control Interno Coso 2013 sobre la gestión de inventario}

El Marco Integrado de Control Interno COSO 2013 ofrece las pautas necesarias, en términos de regulación, que actúan como referente para las organizaciones y potencian el desempeño eficiente de sus procedimientos. En él, se establecen tres divisiones de objetivos que orientan y ayudan a las entidades a enfocarse en los aspectos más importantes relacionados con el control interno: operativos, de información y de cumplimiento. A la par, se establecen cinco componentes integrados con relación a los objetivos mencionados: entorno de control, evaluación de riesgos, actividades de control, sistemas de información y comunicación y actividades de monitoreo y supervisión.

A la vista de esta observación, se observa el vínculo existente entre los objetivos de la organización, los componentes y la estructura de administración. De forma tal que, se introducen dentro de los cinco componentes, un total de diecisiete principios, que abarcan los criterios esenciales y aplican para cada uno de los objetivos mencionados. Los principios descritos, permiten evaluar el sistema de control interno y al mismo tiempo estimar su efectividad. Por lo tanto, las contribuciones anteriores han hecho del Marco Integrado de Control Interno COSO 2013, un modelo robusto y trascendental.

Así, la aplicación del mismo Marco sobre el proceso de gestión de inventarios se hace necesaria e imprescindible para su desempeño eficiente. El mismo, permite a las instituciones eficientizar la gestión del inventario, a través del control adecuado sobre los productos o mercadería, impulsa la obtención de los objetivos de negocio, así como, beneficios económicos y sociales. Lo anterior aportará, además: credibilidad y confiabilidad a la organización, entre otros resultados positivos reflejados en la variable cuota de mercado.

Por tanto, es importante que las organizaciones mantengan una revisión continua sobre los procedimientos de control interno, para mitigar las deficiencias y evitar así, situaciones de fraudes y riesgos, asociadas no solo al inventario. De ahí que, el personal deba estar informado al respecto de los objetivos y metas propuestos para mantener un control interno adecuado, salvaguardar la documentación y toda la información relacionada con la mercadería y los activos de la entidad por el período de tiempo establecido. Así mismo, destacan las siguientes actividades de control: contrastar 
periódicamente la información digital con la plasmada en la documentación física, facilitar y garantizar la comunicación entre las áreas de la entidad, supervisar sistemáticamente las operaciones relacionadas con el almacén, de forma que se acredite el cumplimiento de las regulaciones establecidas.

Sobre lo anterior, se recomienda realizar de forma periódica el conteo de inventario para mantener el control sobre las existencias, evitar costos adicionales por el almacenamiento innecesario de productos obsoletos y mantener el equilibrio sobre los períodos de rotación y en caso necesario, determinar las razones exactas por las que no se respeta el período de rotación.

Es evidente entonces, la relevancia de seleccionar el modelo de control interno adecuado, que establezca los procedimientos acertados hacia el desempeño de las funciones de control en la entidad.

\section{MÉTODO}

En el presente trabajo se realiza una investigación exploratoria y descriptiva. De ahí que, la intención sea precisar los elementos teóricos que fundamenten los beneficios que las organizaciones podrían percibir alrededor de la gestión de inventarios, al implementar como referente en sus procedimientos el Marco de Control Interno COSO 2013.

El Marco de Control mencionado, ha sido seleccionado por la repercusión y buenos resultados que ha conseguido en el entorno internacional. Lo anterior se respalda, además, en que según Barrera (2020) las empresas que cotizan en las bolsas de valores de Estados Unidos tienen la obligación de establecer un Marco de Control Interno y se ha precisado que el Marco de Control Interno COSO 2013 es el más utilizado por dichas entidades.

De otro lado, dicho Marco constituye una herramienta práctica, al establecer las pautas que las empresas deben seguir en cuestiones de Control Interno para el logro de sus objetivos, de ahí que, se facilite el desempeño de las medidas de control que mitiguen los riesgos que interfieren en el logro de las metas del negocio. Particularmente como se describe en este caso de estudio, se obtendrán beneficios ineludibles, mediante el empleo de procedimientos de control Interno sobre la administración eficiente de inventario, en apego a lo establecido en el Marco de Control Interno COSO 2013.

\section{RESULTADOS}

El entorno actual inmerso en cadenas globales de valor y competencia imperfecta mantiene a las empresas en la reinvención constante de sus estrategias de negocio, de cara a la obtención de sus objetivos. En ese sentido, las políticas de control interno juegan un rol protagónico, pues ofrecen a las organizaciones los incentivos, pautas y regulaciones necesarias para mitigar los riesgos posibles. Por tanto, descuidar el control de los inventarios, no es una opción, optar por una administración eficiente de los mismos, en cambio, sí lo es, pues no solo evitará costos adicionales, sino también clientes insatisfechos, lo que, en términos de marketing, representa una gran ventaja.

Sobre la base de las consideraciones anteriores, las empresas deben realizar un seguimiento sobre el inventario en tiempo real, que evite un stock de inventario defectuoso, con el objetivo de satisfacer la demanda de forma sostenida. En ese sentido, es importante que se lleve a cabo una planificación anticipada sobre los valores de inventario, demanda y los ciclos de reorden.

Por tanto, como el comportamiento humano naturalmente ignora los riesgos, se subestiman las posibilidades de ocurrencia de los eventos inusuales. Lo anterior, no pasa desapercibido en el desarrollo e implementación de estrategias de control. Así mismo, las cadenas de suministro, a pesar de los esfuerzos por evitarlo, no siguen un patrón constante, ¿por qué?, porque existe la exogeneidad, y ante contingencias, las medidas tomadas, son por excelencia camaleónicas, en el proceso de acoplamiento o convergencia hacia la coyuntura. Sin embargo, las acciones tomadas deben ofrecer una respuesta responsable que disminuya la exposición prolongada al entorno de incertidumbre y riesgo.

En ese mismo sentido, la presente investigación exploratoria y descriptiva, indica que el control interno favorece la 
gestión de inventarios. Lo anterior, contribuye a mitigar los riesgos por conceptos de: nivel de incertidumbre, infrautilizaciones, ineficacia administrativa, tiempo de espera entre procesos, buffers de protección y exceso de movimientos.

\section{CONCLUSIONES}

Es importante comprender que alrededor de las organizaciones acarrean muchos riesgos, los cuales no se diluyen del todo, pero mediante prácticas adecuadas de control interno, se identifican y se administran. Para enfrentar los riesgos respecto a la gestión de inventarios, estos deben ser categorizados para determinar su importancia en el ciclo de abastecimiento y atención de la demanda al respecto de la cadena de suministro.

Por tanto, los riesgos de la administración de inventarios se encuentran correlacionados con las estrategias dirigidas a definir las funciones de los eslabones de la cadena de suministro, así como el control que sobre ellos se ejerza. Así mismo, es necesario controlar eficientemente el proceso de administración de materiales, la frecuencia de reposición y la determinación de los niveles óptimos de stock, para satisfacer la demanda. Lo anterior, terminará por evitar la tendencia creciente hacia el facilismo de incrementar la cobertura, la cual aumenta la probabilidad de mantener capital inmovilizado, así como la ocurrencia de vencimientos y deterioros de mercancías.

En concordancia con los planteamientos anteriores, los riesgos pueden mitigarse mediante el empleo de controles preventivos y/o mecanismos de control interno que así lo permitan, como es el caso del Marco de Control Interno COSO 2013. Los procedimientos propuestos por el marco en cuestión permitirán a las organizaciones derivar eficientemente las causas y consecuencias de los riesgos, así como la elaboración de planes alternos de ejecución frente a contingencias. Adicionalmente, los riesgos deberán ser monitoreados en tiempo real, para facilitar la toma de medidas preventivas y coordinadas, involucrando las áreas necesarias, ya sean internas o externas a la organización. Todo lo anterior, con la intención de satisfacer oportuna y sosteniblemente la demanda, pese a que la misma está expuesta a factores exógenos.

Ahora bien, ya se mencionaba la exposición de la demanda a la exogeneidad, sin embargo, es posible interpretar la necesidad del cliente en una medida de incertidumbre tolerable, conforme al incremento proporcional percibido en el nivel de servicio. Por tanto, en este escenario, asumiendo un incremento marginal estable (pendiente) y considerando la incertidumbre de la capacidad de abastecimiento de la cadena, ha de mantenerse más alta la disponibilidad de inventario para atender el incremento en la demanda.

Finalmente se concluye que, en la actualidad, las buenas prácticas sobre la administración de inventarios son primordiales para las empresas, ya que se estaría protegiendo una de las mayores inversiones de la organización. Es evidente entonces, que el inventario pueda considerarse capital en forma de productos, aquel que permite a la empresa cumplir con las exigencias de la demanda y competir por elevar su cuota de mercado. 


\section{REFERENCIAS}

Barrera, J. L. (2020). Control Interno, su vínculo con la Eficiencia Operativa y la Rentabilidad. 734-741.En

http://www.web.facpya.uanl.mx/vinculategica/Vinculategica6_1/57\%20BARRERA.pdf

Committee of Sponsoring Organizations of the Treadway Commission. (2013). Internal ControlIntegrated Framework (American Institute of Certified Public Accountants (ed.); Issue May, 2013)

Cruz, A. (2018). Gestión de Inventarios. Editorial. En: https://books.google.es/books?hl=es\&lr=\&id=s1cpEAAAQBAJ\&oi=fnd\&pg=PT8\&dq=Ge $\mathrm{sti} \% \mathrm{C} 3 \% \mathrm{~B} 3 \mathrm{n}+\mathrm{de}+\mathrm{Inventarios} \& \mathrm{ts}=\mathrm{mCdhmO} 41-\mathrm{z} \& \operatorname{sig}=\mathrm{tW}-37-$

2wusrHuLQ1jeH20aBB0lM\#v=onepage $\& q=$ Gesti\%C3\%B3n\%20de\%20Inventarios\&f=fal se

Lizarazo, J. E., \& Perez, E. J. (2017). Aplicación de teorías de inventarios: modelo de suministro de medicamentos para "la caja de previsión de la universidad de cartagena. Universidad de Cartagena. Colombia.

Lopes, I., \& Gómez, M. I. (2012). Auditoría logística para evaluar el nivel de gestión de inventarios en empresas. Ingeniería Industrial/ISSN 1815-5936/Vol. XXXIV/No.1 enero-abril/2013/. 108-118. En: https://www.redalyc.org/pdf/3604/360433593011.pdf

López, A. L. (2011). Control interno al ciclo de inventarios y su impacto en la rentabilidad de la ferretería Ángel López. Universidad Técnica de Ambato. Ecuador.

Mantilla, S. (2018). Auditoría del Control Interno. Editorial. En: https://www.ecoeediciones.com/wp-content/uploads/2018/04/Auditori\%CC\%81a-delControl-Interno-4ed.pdf

Meana, P. P. (2017). Gestión de Inventarios. Editorial. En: https://books.google.com.pe/books?id=M15IDgAAQBAJ\&printsec=frontcover\#v=onepage $\& \mathrm{q} \& \mathrm{f}=$ false

Mora, L. (2016). Gestión logística integral: las mejores prácticas en la cadena de abastecimiento. Ecoe Ediciones, 2016. 354 p. En: https://corladancash.com/wpcontent/uploads/2018/11/Gestion-logistica-integral_-Las-Luis-Anibal-Mora-Garcia.pdf

Morocho, R. L. (2015). La gestión del control de los inventarios y su incidencia en la rentabilidad de la empresa. Universidad Técnica de Machala. Ecuador.

Ortiz, L. M. (2016). Control Interno. COSO 2013 - MICI. En: http://asm.gob.mx/wpcontent/uploads/2017/06/CONTROL_INTERNO_JUNIO_2016.pdf

Pérez, I., Cifuentes, A. M., Vásquez, C., \& Ocampo, D. M. (2013). Un modelo de gestión de inventarios para una empresa de productos alimenticios. Ingeniería Industrial/ISSN 18155936/ vol. XXXIV/No.2 mayo-ago/2013\% En: http://scielo.sld.cu/scielo.php?script=sci_arttext\&pid=S1815-59362013000200009

Rivas, G. (2011). Modelos contemporáneos de control interno. Fundamentos teóricos. Observatorio Laboral Revista Venezolana, vol. 4, núm. 8, julio-diciembre, 115-136. En: https://www.redalyc.org/pdf/2190/219022148007.pdf

Taha, H. A. (2012). Investigación de Operaciones. Pearson. Editorial. En: https://jrvargas.files.wordpress.com/2009/01/investigacic3b3n-de-operaciones-9naedicic3b3n-hamdy-a-taha-fl.pdf 